\title{
Anomalous Scattering and Null-Domain Ghost Corrections for Fibre Textures
}

\section{FUENTES} Center of Applied Research For Nuclear Development P.O. Box 6122 Havana,
Cuba

(Received September 14, 1987; in final form August 5, 1988)

\begin{abstract}
The practical applicability of anomalous scattering and null-domain ghost corrections for fibre textures is theoretically evaluated. For a hypothetical asymmetric orientation distribution of quartz-like $\mathrm{BPO}_{4}$ highly absorpting crystals, slightly asymmetric anomalous scattering pole figures are predicted. On the basis of projection relations among orientation distribution functions and inverse pole figures, the special characteristics of the null-domain method for fibre textures are discussed, with the suggestion of a practical procedure to estimate (in favourable cases) an upper limit for ghosts effects.
\end{abstract}

KEY WORDS: Fibre textures, neutron diffraction, anomalous scattering, parity, ghost correction, $\mathrm{BPO}_{4}$.

\section{INTRODUCTION}

The study of ghost phenomena constitutes an important part of texture analysis. The majority of the publications, at present, are devoted to the development of methods to evaluate- or at least to estimate-the odd part of the Orientation Distribution Function (ODF) in three-dimensional textures (Bunge, Esling, Muller 1980, Wagner et al. 1981, Matthies and Helmig 1982, Helmig and Matthies 1984, Feldmann and Fuentes 1983).

Here some special characteristics of the so-called fibre textures are discussed in relation to this problem. First, three-dimensional crystal orientations and fibre textures are compared with respect to the "parity" of their mathematical descriptions. Then, slightly asymmetric anomalous scattering pole figures are predicted for a 
hypothetical extremely asymmetric fibre texture. Finally the capabilities of the null-domain method for the estimation of ghost effects in fibre textures are discussed.

Quartz-like polycrystals, as considered by Feldmann and Fuentes 1983 shall be used as the concrete observation system. It consists of non-centrosymmetrical crystals, with atoms giving rise to strong anomalous scattering effects.

\section{PARITY OF THE TEXTURE FUNCTIONS}

The analytical representation of fibre textures and the one corresponding to general three-dimensional orientation distributions differ in the character of the "parity" of its even and odd parts.

In three-dimensional textures the ODF admits no evaluation of parity, because the inversion operation has no meaning for orientations. The names "even" and "odd" given respectively to the components $\tilde{f}(g)$ and $\tilde{\tilde{f}}(g)$ of the ODF express the parity of the index $l$ in the corresponding sums of the generalized spherical harmonics $\left(T_{l}^{m n}\right)$ expansion of the ODF $f(g)$, but the so-named functions are neither even nor odd in the sense defined e.g. by Landau and Lifshits 1965. Mathematically

$$
\begin{aligned}
f(g) & =\sum_{l=0(1)}^{\infty} \sum_{m=-l}^{l} \sum_{n=-l}^{l} C_{l}^{m n} T_{l}^{m n}(g) \\
& =\tilde{f}(g)+\tilde{\tilde{f}}(g)
\end{aligned}
$$

with:

$$
\begin{aligned}
& \tilde{f}(g)=\sum_{l=0(2)}^{\infty} \sum_{m} \sum_{n} C_{l}^{m n} T_{l}^{m n}(g) \\
& \tilde{f}(g)=\sum_{l=1(2)}^{\infty} \sum_{m} \sum_{n} C_{l}^{m n} T_{l}^{m n}(g)
\end{aligned}
$$

The ODF cannot be negative, but this does not imply any restriction on the sign of $\tilde{f}(g)$.

Fibre textures, on the other hand, can be represented by Inverse Pole Figures (IPF) and these may indeed possess parity, in the common sense. The IPF is a projection of the ODF, obtained by 
integrating $f(g)$ in all the orientations of the Euler space for which the crystal direction $h$ is parallel to the sample direction $y$ (assuming constant $\mathbf{y})$. The IPF $R(\mathbf{h})$ so obtained admits comparison with $R(-h),-h$ being opposite to $h$ and $R(-h)$ consisting of the integral of $f(g)$ for all directions $\mathbf{h}$ antiparallel to $\mathbf{y}$.

In the spherical harmonics $\left(Y_{l}^{m}\right)$ expansion of $R(\mathbf{h})$ :

$$
\begin{aligned}
R(\mathbf{h}) & =\sum_{l=0(1)}^{\infty} \sum_{m=-l}^{l} C_{l}^{m} Y_{l}^{m}(\mathbf{h}) \\
& =\tilde{R}(\mathbf{h})+R(\mathbf{h}) \geqslant 0 \\
\tilde{R}(\mathbf{h}) & =\sum_{l=0(2)}^{\infty} \sum_{m} C_{l}^{m} Y_{l}^{m}(\mathbf{h}) \\
\tilde{R}(\mathbf{h}) & =\sum_{l=1(2)}^{\infty} \sum_{m} C_{l}^{m} Y_{l}^{m}(\mathbf{h})
\end{aligned}
$$

as a consequence of

$$
Y_{l}^{m}(-\mathbf{h})=(-1)^{l} Y_{l}^{m}(\mathbf{h})
$$

$\tilde{R}$ is an even function and $R$ is odd. From this it follows, as has been pointed out by Matthies (1980) that $\tilde{R} \geqslant 0$, always.

The mentioned difference in relation to the parity of the ODF and the IPF has its implications in the representation of textures: ODFs require the same irreducible region in Euler space for $f(g)$ as it does for its "even" part; IPFs need only a half domain for $\tilde{R}$ as compared with that for $R$. In the following, the irreducible regions for $R$ and $\tilde{R}$ will be referred to as "unreduced" and "reduced", respectively. At the border of the reduced region $\tilde{R}=0$.

\section{MODEL FIBRE TEXTURES FOR QUARTZ-LIKE STRUCTURES}

Now the hypothetical system that constitutes the object of interest is presented.

Consider the ceramic $\mathrm{BPO}_{4}$, crystallizing in a quartz-like structure, as reported by Dachile and Glasser 1959. The symmetry of this structure is trigonal, with point group $D_{3}$. Let the polycrystal sample contain only one sort (right- or left-handed) of enantiomorphic crystals, with only ${ }^{10} \mathrm{~B}$ atoms. 
Figures 1 and 2 show the IPFs of two model fibre textures for this system. They are remarkable for the relative weights of their even and odd components.

The distribution of Figure 1 shows a Gaussian peak with maximum intensity of 8.6 at $(1,1, \overline{2}, 1)$. It's half-width is $w=13^{\circ}$. It has been computer-generated according to the function

$$
R=R_{0} e^{-0.693(\Omega / w)^{2}}
$$

where $\Omega$ is the angle between the observation point and the center of the peak, $w$ is the half-width and $R_{0}$ is the maximum intensity. The considered distribution was built on the assumption of a ratio of $10: 1$ between the intensities at $(1,1, \overline{2}, 1)$ and at $(1,1, \overline{2}, 0)$. The

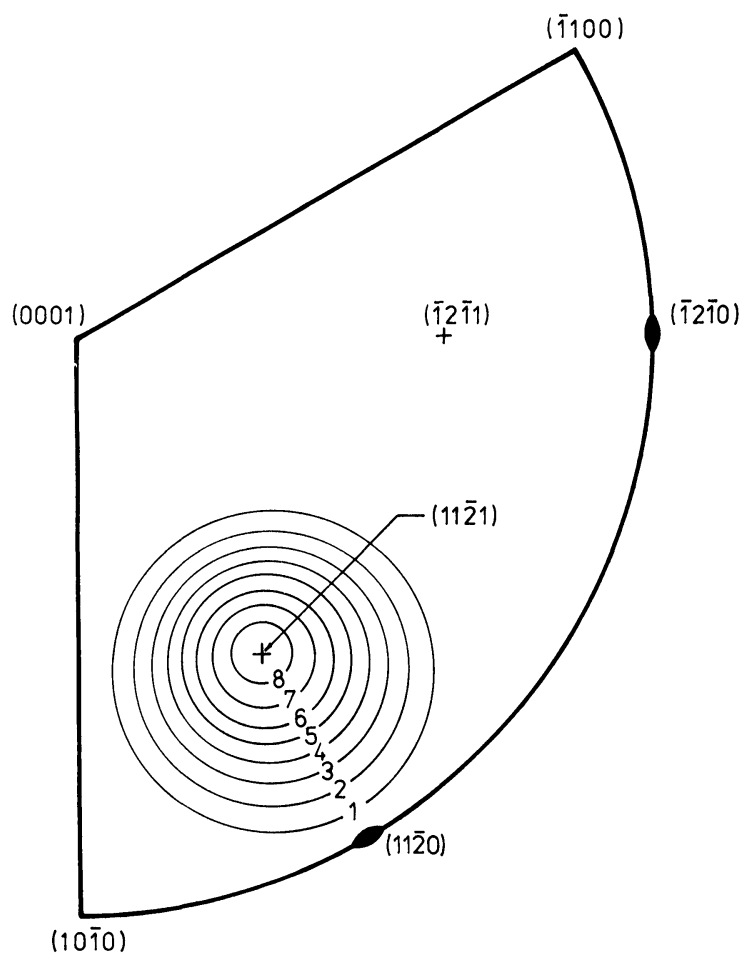

Figure 1 Fibre axis inverse pole figure (IPF) of a hypothetical polycrystal of $\mathrm{BPO}_{4}$. The texture has one Gaussian-like component at $(1,1, \overline{2}, 1)$ with maximum intensity 8.60 and half width $13.4^{\circ}$. 


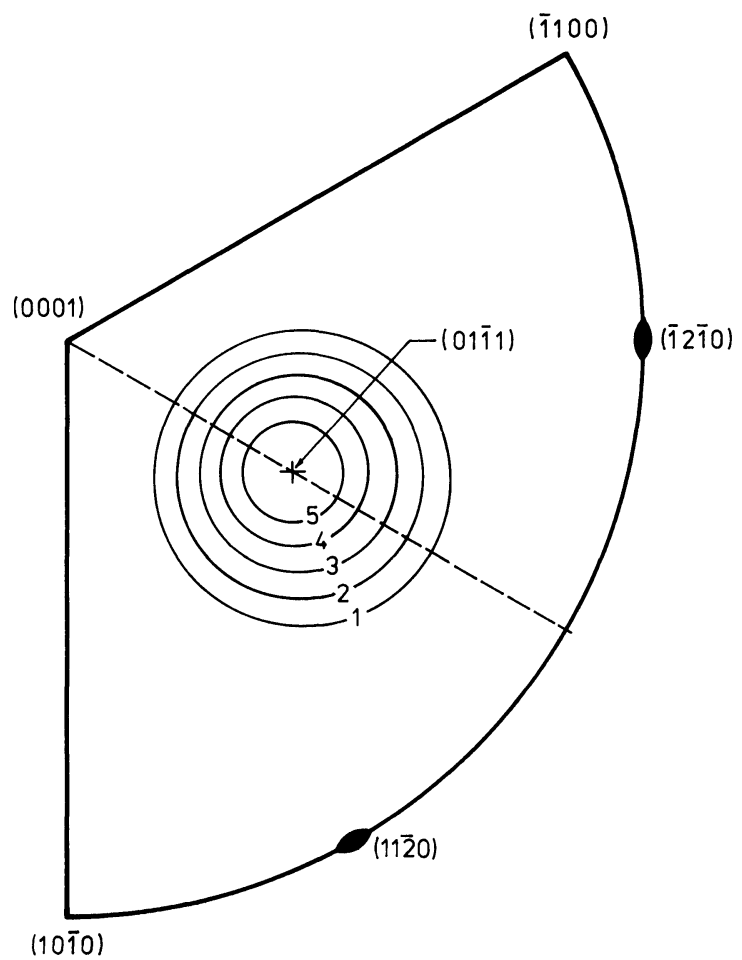

Figure 2 Inverse pole figure of an "even" fibre texture. The discontinuous line represents a mirror plane of symmetry for the considered distribution.

maximum intensity has been so chosen as to make the distribution normalized to $4 \pi$ for its surface integral over the whole projection sphere (considering multiplicity, as below).

If this IPF was discomposed into its even and odd parts, then $\tilde{R}$ would show two peaks in the unreduced region: one at $(1,1, \overline{2}, 1)$ and other at $(\overline{1}, 2, \overline{1}, 1)$, each with maximum intensity 4.3 . $\tilde{\tilde{R}}$ would have a positive peak, equal to that of $\tilde{R}$, at $(1,1, \overline{2}, 1)$. At $(\overline{1}, 2, \overline{1}, 1)$ it would show a negative peak, with intensity -4.3 , such that $\tilde{R}+\tilde{R}=0$ in the whole region around $(\overline{1}, 2, \overline{1}, 1)$.

The texture corresponding to Figure 2 was built up by the same algorithm as that of Figure 1, but with its peaks located in such a way as to give a centrosymmetrical distribution. This pure "even" 
IPF shows a maximum intensity of 6.0 at $(0,1, \overline{1}, 1)$ and a half width $w=16.0^{\circ}$. As the inversion operation is effective, the texture point group is $D_{3 d}$ and the discontinuous line bisecting the unreduced triangle represents a mirror plane.

The multiplicity over the projection sphere of both configurations is 6 . The locations of the respective maxima are the following.

- Texture of Figure 1:

$$
\begin{array}{lll}
(1,1, \overline{2}, 1) ; & (1,1, \overline{2}, \overline{1}) ; & (\overline{2}, 1,1,1) ; \\
(\overline{2}, 1,1, \overline{1}) ; & (1, \overline{2}, 1,1) ; & (1, \overline{2}, 1, \overline{1})
\end{array}
$$

\section{- Texture of Figure 2:}

$$
\begin{array}{lll}
(0,1, \overline{1}, 1) ; & (1,0, \overline{1}, \overline{1}) ; & (\overline{1}, 0,1,1) ; \\
(\overline{1}, 1,0, \overline{1}) ; & (1, \overline{1}, 0,1) ; & (0, \overline{1}, 1, \overline{1}) .
\end{array}
$$

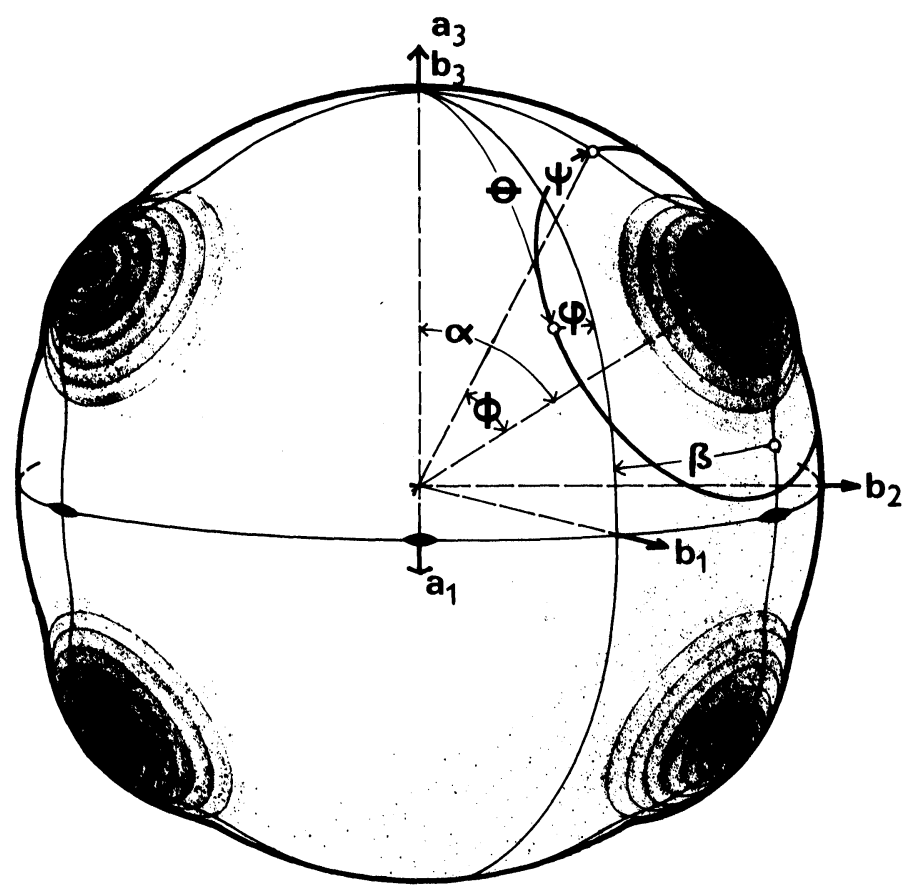

Figure 3 Surface representation of the complete IPF corresponding to the texture represented in Figure 1. To determine the $h_{0}=(1,1, \overline{2}, 1)$ unreduced pole figure, the Fundamental Equation for Fibre Textures is used. Angles are defined in the text. 
A surface representation of the texture associated to Figure 1, with its multiplicity taken into account, is given in Figure 3. Four of the six components of this configuration are shown. Direct $\left(a_{i}\right)$ and reciprocal $\left(\mathbf{b}_{j}\right)$ fundamental vectors are represented.

\section{ANOMALOUS SCATTERING}

The possibility of ghost-corrections by means of anomalous scattering of X-rays or neutrons has been theoretically established by Bunge and Esling (1981). The particular case of quartz-like structures was evaluated by Feldmann and Fuentes (1983). Here the calculations of the latter authors are extended to predict the characteristics of the pole figures that would be measured in a neutron anomalous scattering experiment, in the case of the fibre symmetries presented in the preceding section.

The "unreduced" or "structural" pole figures associated to a given IPF are given by the so-called Fundamental Equation for Fiber Textures (Bunge 1982)

$$
P_{\mathrm{h}_{0}}(\phi)=\frac{1}{2 \pi} \int_{0}^{2 \pi} R(\phi, \psi) d \psi
$$

Figure 3 shows the details of the calculation of $P_{h_{0}}(\phi)$ in the specific case in which $\mathbf{h}_{0}=(1,1, \overline{2}, 1)$ for the texture of Figure 1 . $P_{h_{0}}(\phi)$ is the pole density at angle $\phi$, and the integral averages the IPF $R(h)$ along a circumference located at an angular distance $\phi$ from the pole $\mathbf{h}_{0}$.

The angular coordinates of the observation point $\mathbf{h}$ are usually given as the polar and azimuthal angles $(\theta, \varphi)$ measured from the "north pole" and a reference "meridian" of the projection sphere, respectively. To perform the integral in (11), trigonometric formulas which relate the angular coordinates, the distance $\phi$ and the scanned angle $\psi$ are needed. For the pole figure $\mathbf{h}_{0}=(\alpha, \beta)$ these can be represented as:

$$
\begin{gathered}
\cos \theta=\cos \alpha \cos \phi+\sin \alpha \sin \phi \cos \psi \\
\cos (\beta-\varphi)=\frac{\cos \phi}{\sin \theta \sin \alpha}-\operatorname{ctg} \theta \operatorname{ctg} \alpha
\end{gathered}
$$




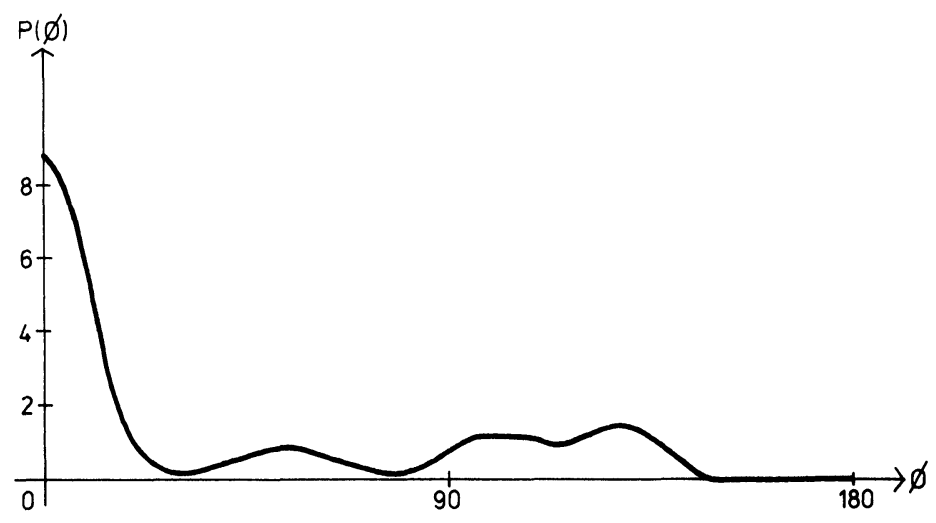

Figure 4 Unreduced $(1,1, \overline{2}, 1)$ pole figure corresponding to the texture of Figures 1 and 3.

In general, the integral (11) should be evaluated for $0<\phi<\pi$. For the considered case, the result of these calculations is presented in Figure 4, in which the asymmetry of $P_{h_{0}}(\phi)$ with respect to $\phi=\pi / 2$ is remarkable. This is a consequence of $R$ being null at and around $(\overline{1}, \overline{2}, 2, \overline{1})$.

Figure 4 gives the actual population of the considered crystallographic planes at any angle from the symmetry axis of the sample, but it is not what would be measured in a diffraction experiment. A diffraction experiment superposes the pole figures corresponding to all the families of planes having the same interplanar distances " $d$ ", whether they are crystallographically equivalent or not.

This question has been analyzed by Bunge and Wenk in relation to the permutation of the indices $h$ and $k$ in the $(h, k, i, l)$ pole figures of quartz (Bunge and Wenk, 1977), and by Bunge and Esling (1981) in the discussion of unreduced pole figure measurement by anomalous scattering in general, the pole figure that is obtained in a diffraction experiment is:

$$
\hat{P}_{\mathrm{h}_{0}}(\phi)=\sum_{n=1}^{N} a_{n} P_{n}(\phi)
$$

where $N$ is the number of families of planes having the same " $d$ ", but different structure factors $|F|^{2}, a_{n}$ is proportional to $|F|^{2}$ and 
such that:

$$
\sum_{n} a_{n}=1
$$

Considering the anomalous scattering of neutrons, it is possible to predict the $(1,1, \overline{2}, 1)$ pole figure that would be measured over the sample of Figure 1 . In this case $N=2$. The weight factors to be used are (see Feldmann and Fuentes 1983)

$$
\begin{gathered}
a(1,1, \overline{2}, 1)=0.558 \\
a(\overline{1}, \overline{1}, 2, \overline{1})=a(\overline{1}, 2, \overline{1}, 1)=0.442
\end{gathered}
$$

The result of these calculations is shown on Figure 5. The interesting point here is the asymmetry of the pole figure, as may be characterized by the difference $\hat{P}_{h}(\phi)-\hat{P}_{h}(\pi-\phi)$. The maximum of this variation occurs for $\phi=0^{\circ}$ and $180^{\circ}$, and has a relative value of:

$$
\frac{\hat{P}_{h_{0}}\left(0^{\circ}\right)-\hat{P}_{h_{0}}\left(180^{\circ}\right)}{\frac{1}{2}\left[\hat{P}_{h_{0}}\left(0^{\circ}\right)+\hat{P}_{h_{0}}\left(180^{\circ}\right)\right]}=23 \%
$$

This figure characterizes the small effect that would be detected in a good experiment over such a sample.

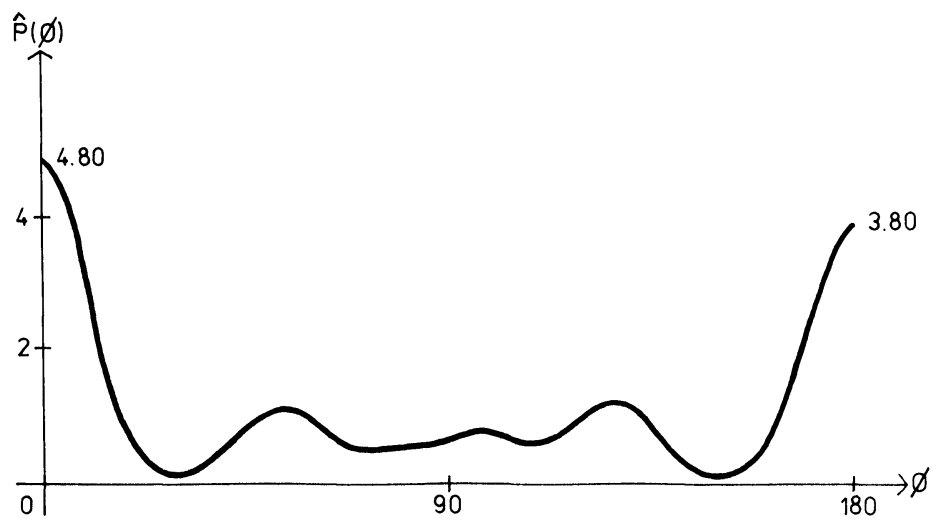

Figure 5 "Anomalous scattering" pole figure of the texture of Figure 1. The strong asymmetry represented in the IPF by the absence of a peak at $(\overline{1}, 2, \overline{1}, 1)$ is weakly projected here as a difference of $23 \%$ between $\hat{P}\left(0^{\circ}\right)$ and $\hat{P}\left(180^{\circ}\right)$. 
With the same sample of Figure 1, the following anomalous scattering pole figures would also be asymmetric with respect to $\phi=\pi / 2$ :

$$
(1,1, \overline{2}, 0) ; \quad(1,1, \overline{2}, 2) ; \quad(2,1, \overline{3}, 1) ; \quad(1,1, \overline{2}, 3) ; \quad(2,1, \overline{3}, 3)
$$

while the following would not present asymmetry:

$$
\begin{array}{lll}
(1,0, \overline{1}, 0) ; & (1,0, \overline{1}, 1) ; & (1,0, \overline{1}, 2) ; \\
(2,0, \overline{2}, 1) ; & (2,0, \overline{2}, 3) ; \quad(1,0, \overline{1}, 4)
\end{array}
$$

If anomalous scattering was not present, then the values $a_{n}=1 / 2$ would hold for $n=1,2$, and the so-called reduced pole figures would be measured, according to:

$$
\tilde{P}_{\mathbf{h}_{0}}(\phi)=\frac{1}{2}\left[P_{\mathbf{h}_{0}}(\phi)+P_{-h_{0}}(\phi)\right]
$$

The reduced $(1,1, \overline{2}, 1)$ pole figure that would be so obtained is presented here as Figure 6 . The symmetry of this figure is a consequence of Friedel's law. If this and analogous pole figures were taken as experimental data for calculating the IPF, the result would be the $\tilde{R}$ mentioned in the presentation of the considered

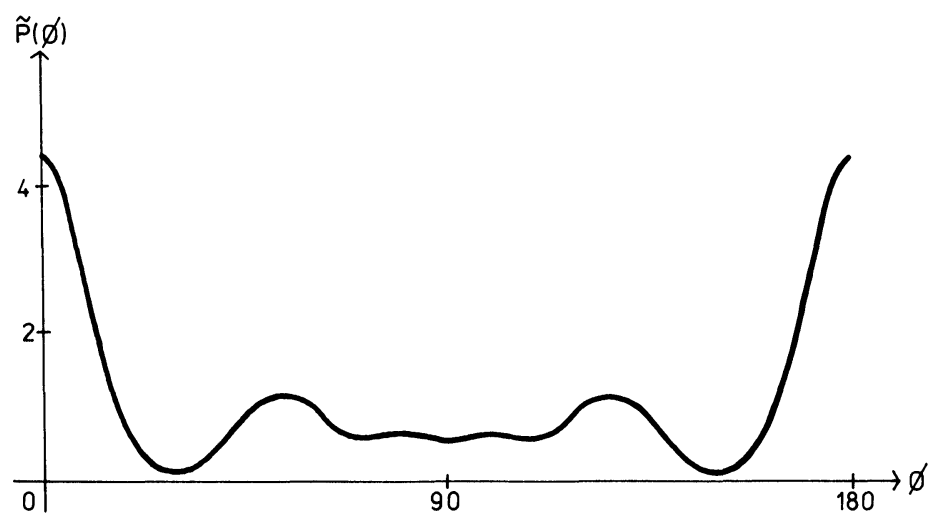

Figure 6 Reduced $(1,1, \overline{2}, 1)$ pole figure associated to the texture of Figure 1. This is the experimental result to be obtained by normal diffraction from the considered texture. The IPF to be deduced from this and analogous curves would be composed of two texture peaks, each with maximum intensity 4.30 , located at $(1,1, \overline{2}, 1)$ and $(\overline{1}, 2, \overline{1}, 1)$. 
model texture. So one would obtain a "ghost texture peak" at $(\overline{1}, 2, \overline{1}, 1)$.

In a typical experiment, right- and left-handed crystals would be present, each sort having its orientation distribution. If both sorts showed the considered texture, then the anomalous scattering pole figures would have a form between the limits of Figures 5 and 6, depending on the relative concentrations. For the case of equal concentrations and orientation distributions the experimental result would be symmetric like that of Figure 6 .

With the texture of Figure 2, as a consequence of $R(-\mathbf{h})=R(\mathbf{h})$ for all $h$, all the anomalous scattering pole figures would be symmetric with respect to $\phi=\pi / 2$.

Taking into account the special characteristics of the considered hypothetical samples, Figure 5 could be regarded as an optimistic limit for the capabilities of the anomalous scattering technique for ghost texture corrections.

\section{NULL DOMAINS}

The non-negativity condition of the ODF, applied to the so-called null domains, permits low order corrections of ghost phenomena, as has been established by Bunge et al. (1980) for three dimensional textures. In fibre textures the null domain treatment should adopt a particular form, in correspondence with the special characteristics of the situation.

Consider, first, the case represented in Figure 1. This texture has a large null domain, virtually the whole reduced region around the $(\overline{1}, 2, \overline{1}, 1)$ pole. This null domain would not be detectable in a normal diffraction experiment for, as was discussed in the preceding section, only symmetric pole figures would be measured and these would imply a ghost texture peak at the just mentioned pole. Thus for such a texture the null domain method would be practically useless.

Now the case associated to Figure 2 is analyzed. A normal diffraction study of such a texture would lead just to the "even" distribution shown in this Figure, but the consideration of ghost phenomena would imply some uncertainty as to the reality or not of the symmetry represented by the mirror plane bisecting the 
unreduced triangle of the IPF. Mathematically, $\tilde{\tilde{R}}$ would not be known, and an estimate of its possible effect on $R$ would be needed.

The properties of $R, \tilde{R}$ and $\tilde{R}$ permit the evaluation of a plausible upper limit for $\tilde{R}$ as a characterization of the uncertainty associated to the IPF.

Searching for a top for $\tilde{R}$, let it be represented as a superposition of positive and equal-but-negative Gaussian bells, distributed symmetrically at both sides of the bisecting plane in Fig. 2. Any negative peak of $\tilde{\tilde{R}}$ is limited in its intensity by the condition $\tilde{R}+\tilde{\tilde{R}} \geqslant 0$. Assuming that $\tilde{\tilde{R}}$ is smooth, it should adopt its maximum values at points far from the border of the reduced region, where the condition $\tilde{R}=0$ is satisfied.

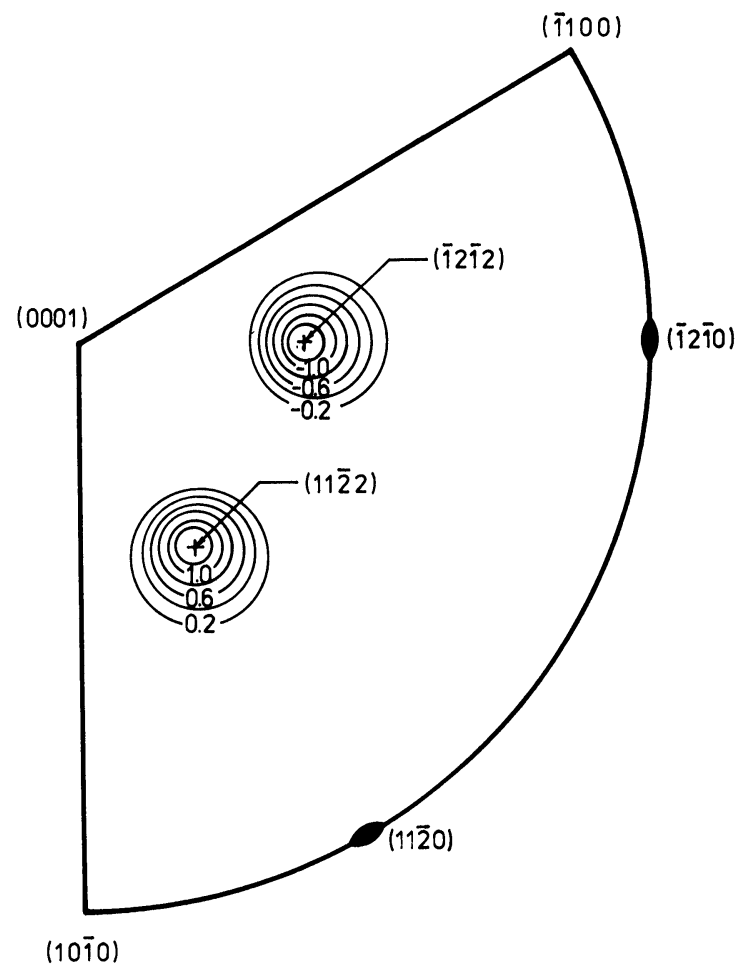

Figure 7 An estimate of the upper limit to the admissible "odd part" for the texture of Figure 2. 
With this points in mind, the following composition for $\tilde{\tilde{R}}$ is proposed: a positive peak with its maximum in the central part of the lower half of the unreduced triangle of Figure 2, and a symmetric but negative peak in the corresponding region of the upper half of this figure. This function, with its maximum limited by the condition (6), gives a coarse estimate of the most intense odd component $\tilde{\tilde{R}}$ that shows a simple structure and is compatible with the given even component $\tilde{\tilde{R}}$. To define a specific shape for the proposed bells, Ec. (10) is proposed. As "centers of the reduced regions" the poles $(1,1, \overline{2}, 1)$ and $(\overline{1}, 2, \overline{1}, 2)$ are for simplicity chosen. To assign a maximum intensity to these odd components, $\tilde{R}$ is evaluated at $(1,1, \overline{2}, 2)$ and then $\tilde{R}(1,1, \overline{2}, 2) \equiv$

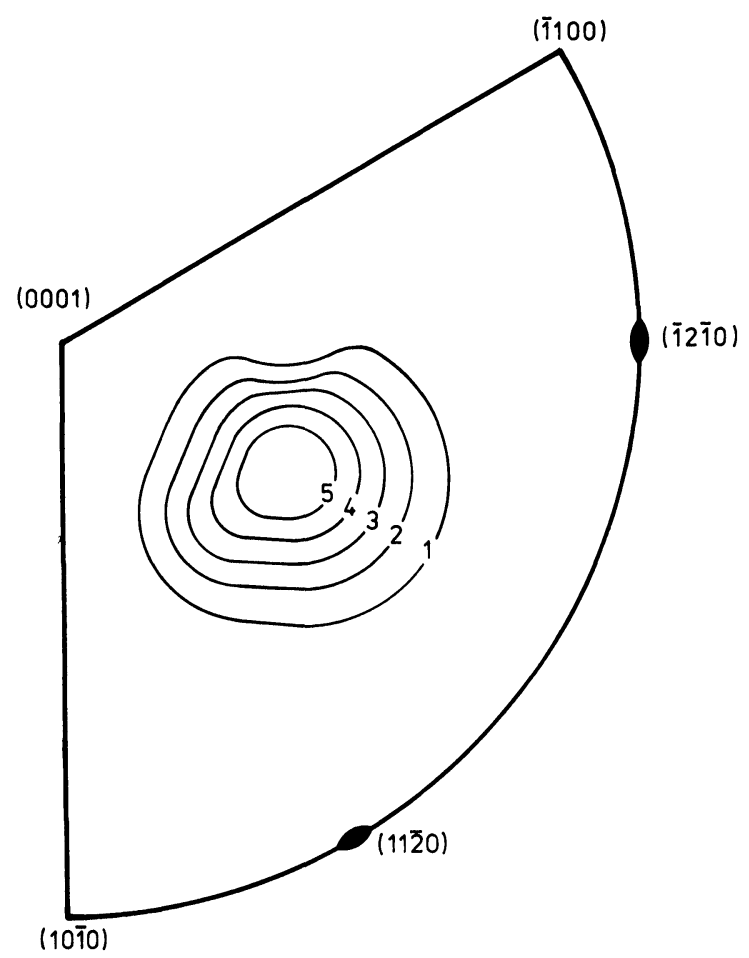

Figure 8 Superposition of the IPFs associated to Figs. 2 and 7. This drawing gives a crude representation of the possible distorting effect of ghost phenomena on the texture of Figure 2. 
$-\tilde{\tilde{R}}(\overline{1}, 2, \overline{1}, 2) \equiv \tilde{R}(1,1, \overline{2}, 2)$ are defined. The width of the $\tilde{\tilde{R}}$ bells is chosen similar to that of $\tilde{R}$.

For the specific case of Figure 2, the parameters for $\tilde{R}$ are the following:

$$
\tilde{R}(1,1, \overline{2}, 2)=-\tilde{\tilde{R}}(\overline{1}, 2, \overline{1}, 2)=1.4
$$

Figure 7 shows the $\tilde{R}$ so obtained. The superposed IPF $R=\tilde{R}+\tilde{\tilde{R}}$ is presented in Figure 8. This last figure gives a crude representation of the possible distorting effect of ghost phenomena on the considered texture.

In general, $\tilde{\tilde{R}}$ should not be more disturbing than a surface, similar to that of Figure 7, with maximum intensity given by $|\tilde{R}|=\tilde{R}$ at the centre of the reduced region of the projection sphere. Sharp textures, with peak intensities at the border of this region, should not be severely affected by ghost phenomena.

\section{Acknowledgements}

The ideas for the present work were born during interesting discussions held at JINR, Dubna, with Dr. K. Feldmann and Dr. K. Walther. Their technical and moral support is gratefully acknowledged.

\section{References}

Bunge, H. J. (1982) Texture Analysis in Materials Science. Butterworths Publ. London, p. 126.

Bunge, H. J. and Esling, C. (1961) J. Appl. Cryst. 14, p. 253-255.

Bunge, H. J., Esling, C. and Muller, J. (1980) J. Appl. Cryst. 13, p. 544-554.

Bunge, H. J. and Wenk, H. R. (1977) Tectonophysics 40, p. 257-285.

Dachile, F. and Glasser, L. S. D. (1959) Acta Cryst. 12, p. 820-821.

Feldmann, K. and Fuentes, L. (1983) Preprint E 14-83-697, JINR, Dubna.

Helmig, K. and Matthies, S. (1984) Phys. Stat. Sol. (b) 126, p. 43-52.

Landau, L. D. and Lifshits, E. M. (1965) Quantum Mechanics, Non-Relativistic Theory. Pergamon Press London, p. 95.

Matthies, S. (1980) Phys. Stat. Sol. (b) 98 K, p. 11-36.

Matthies, S. and Helmig, K. (1982) Phys. Stat. Sol. (b) 113, p. 569-582.

Wagner, F., Wenk, H. R., Esling, C. and Bunge, H. J. (1981) Phys. Stat. Sol. (a) 67, p. 269-285. 